\title{
Rol del Estado frente al envejecimiento de la población: el caso de Chile
}

\author{
The role of the State before population aging: case of Chile
}

\author{
Francisco Ganga-Contreras / fganga@ulagos.cl \\ Universidad de Los Lagos, Chile \\ María Angélica Piñones-Santana / angelica.pinones@uv.cl \\ Diego González-Vásquez / diego.gonzalez@uv.cl \\ Francisca Rebagliati-Badal / f.rebagliatibadal@gmail.com \\ Universidad de Valparaiso, Chile
}

\begin{abstract}
States must establish the basis for people to age with adequate living conditions in all areas. Therefore, the cardinal purpose of this research is to describe the role played by the Chilean state to face Population Aging, PA, developing for this an analysis that determines the relation between the implemented measures and the objectives of the public policy created for this purpose. To achieve the research purposes, an exploratory-descriptive study was carried out, analyzing the main variables involved in the process and identifying the essential components.

Although actions that seek to face this context have been taken, it is unclear if they respond to defined strategic guidelines or reactive policymaking arising from the delayed response to existing problems, what is clear is that States must act promptly to deal with this present challenge.
\end{abstract}

Key words: population aging, Latin America, Chile, State, public policy.

Resumen: Los Estados deben establecer las bases para que las personas envejezcan con condiciones de vida adecuadas en todos sus ámbitos. Considerando este desafío, se ha planteado como propósito cardinal de la presente investigación describir el papel que ha jugado el Estado chileno, para enfrentar el Envejecimiento Poblacional (EP), desarrollando para ello un análisis que determine la relación entre las medidas implementadas y los objetivos de la política pública creada para este fin. Para lograr los propósitos indagativos, se realizó un estudio exploratorio-descriptivo, analizando las principales variables que conforman el proceso e identificando los componentes esenciales del mismo.

Si bien se han tomado medidas que buscan enfrentar este contexto, no está claro si responden a lineamientos estratégicos definidos o a medidas reactivas surgidas de la respuesta tardía a problemas ya existentes; lo que queda claro es que los Estados deben actuar oportunamente frente a este desafío que se presenta.

Palabras clave: Envejecimiento de la población, Latinoamérica, Chile, Estado, política pública. 


\section{Introducción}

El Estado en su permanente búsqueda de soluciones para el alivio de la pobreza y una mejor distribución del ingreso, se ha visto obligado a modificar y diseñar políticas públicas que ayuden a enfrentar nuevas problemáticas como, por ejemplo, el envejecimiento de la población.

El cambio en la pirámide demográfica mundial y nacional ya se está haciendo notar en los distintos sectores prioritarios para el Estado, como son: salud, educación, pensiones, vivienda, entre otros.

Cuando se habla de envejecimiento, se hace referencia a un proceso de largo camino, con un desarrollo gradual y que se puede visibilizar con antelación. Esto permite que los Estados tengan tiempo para poder determinar los escenarios de acción posibles para enfrentar esta problemática.

Con el paso de los años y con necesidades distintas, cambia la conducta en la demanda de las personas mayores; sus principales deseos y necesidades apuntan a tener un envejecimiento cuya calidad de vida no se distancie de la que poseían en su etapa de vida laboral, evitando el desmedro económico y manteniendo su nivel socioeconómico, permitiéndoles optimizar la misma, entendiendo esto como algo que va más allá de los lujos y el poder adquisitivo. Esta calidad de vida tiene relación más bien con aspectos como la salud, integración social, entre otros.

Los cambios etarios de la población son un tema que se proyecta como un proceso que será constante en los siguientes años y décadas, aumentando la población mayor de 65 años, frente a la población total. Según estudios de la Corporación de Investigación, Estudio y Desarrollo de la Seguridad Social (CIEDESS) (2012) se proyecta que la población con más de 65 años pasará desde cerca de $10 \%$ hasta poco menos de $15 \%$ en 2025 , y a más de $20 \%$ hacia el año 2050.

Claramente, el envejecimiento de la población es más que un cambio demográfico; no es una modificación en las cifras con las que se construyen políticas públicas, sino más bien una transformación en la forma como se desarrolla la sociedad en su conjunto y cómo debe enfrentar su desarrollo futuro.

Es menester considerar que para los Estados el tema del envejecimiento en la población se ha convertido en un desafío, que no se vislumbra fácil de afrontar, entendiendo que éstos deben propender a generar las condiciones necesarias para que las personas puedan desarrollarse en un entorno de calidad, con salud adecuada, vivienda e integrados a la sociedad, entre otros aspectos. 
En este orden de cosas, el presente trabajo se ha planteado como objetivo primordial describir el rol que ha jugado el Estado de Chile, para enfrentar el envejecimiento poblacional. Para ello ha desarrollado un análisis que determine la relación que tienen las medidas implementadas con los objetivos de la política pública creada en este país para dicho fin.

Para alcanzar el propósito central planteado, se utiliza como metodología un enfoque mixto, pues se realiza un proceso de recolección, análisis y vinculación de datos cuantitativos y cualitativos en un mismo estudio o serie de investigaciones para responder a un planteamiento del problema (Hernández y Baptista, 2006: 751). Además, se puede mencionar que es de carácter exploratorio, ya que en este trabajo se examina un tema o problema de investigación poco estudiado previamente (Hernández y Baptista, 2006: 100).

Como se observará, esta indagación debiera contribuir a la discusión de las políticas públicas y estrategias sectoriales relacionadas con los adultos mayores en Chile, su principal aportación es dar a conocer cuáles han sido los programas gubernamentales que ha impulsado este país, para responder al desafío del envejecimiento de la población, y cómo se relacionan con los objetivos enmarcados en la política pública creada por el Estado chileno para dicho fin.

\section{Política pública}

De acuerdo con Aguilar y Lima (2009), en los años cincuenta Harold Lasswell, pionero de la ciencia política, define a la política pública como "disciplinas que se ocupan de explicar los procesos de elaboración y ejecución de las políticas... con base científica, interdisciplinaria y al servicio de los gobiernos democráticos". Veinte años después, en 1971, agregaría un punto crucial en la definición: "el conocimiento del proceso de decisión y el conocimiento en el proceso de decisión”.

Años más tarde las definiciones del concepto de política pública se centran en diversos enfoques. El primer enfoque está centrado en la finalidad de la decisión, respecto de si ésta se orienta a alterar o no el estado de cosas vigentes. Por ejemplo, Kraft y Furlong (2004) señalan que una "política pública es un curso de acción (o inacción) que el Estado toma en respuesta a problemas sociales". Los expertos señalan, a propósito de ello, que las políticas públicas reflejan no sólo los valores más importantes en la sociedad, sino que también muestran el conflicto entre los valores y cuáles de éstos reciben las mayores prioridades en una determinada decisión. 
El segundo enfoque centra su atención en la decisión misma que hace surgir una política pública. Para Jenkins (1978), la política pública hace referencia a un conjunto de decisiones interrelacionadas, tomadas por un actor $\mathrm{o}$ grupo de actores respecto de la selección de metas y medios para alcanzar en una situación específica, y donde aquellas decisiones están dentro del ámbito de autoridad de esos actores. Es decir, "la mayoría de las políticas públicas involucran una serie de decisiones, algunas de las cuales pueden ser inadvertidas antes que deliberadas, pero, acumulativamente, todas estas decisiones constituyen una política pública" (Olavarria, 2007:16).

Un tercer enfoque del concepto centra su atención en la dimensión del poder. Los expertos Meny y Thoening (1992) enfatizan el rol de la autoridad pública, señalando que una política pública corresponde a la acción de una autoridad investida de poder público y de legitimidad gubernamental.

De acuerdo con Birkland (2005), no hay consenso entre los expertos respecto de una definición única de política pública. Pero para términos de esta investigación se entenderá por política pública la definición del experto chileno Eugenio Lahera (2008: 28): “cursos de acción y flujos de información relacionados con un objetivo público definido en forma democrática; dichos cursos de acción o de información son desarrollados por el sector público y, frecuentemente, con la participación de la comunidad y el sector privado".

Según Lahera (2008), las políticas públicas se componen de cinco fases: origen, programa, diseño, gestión y evaluación de políticas públicas. Sin embargo, aclara que dichas "fases" no forman parte de un ciclo que siga una secuencia lineal, debido a dos razones primordiales. En primer lugar, porque tanto los sistemas políticos como administrativos son imperfectos. Y en segundo lugar, porque tanto los datos de la realidad social como económica van cambiando.

Por ello tanto los problemas como las soluciones de una política pública están constantemente redefiniéndose.

En este orden de cosas, es relevante mencionar que las políticas públicas son comúnmente operacionalizadas bajo la forma de programas y proyectos, existiendo pautas o guías para facilitar su implementación (Subsecretaría de Desarrollo Regional y Administrativo [SUBDERE], 2009).

Ahora bien, se entiende por programa, según el Art. 2, núm. 1, Ley 20.530/2011, al "conjunto integrado y articulado de acciones, prestaciones y beneficios destinados a lograr un propósito específico en una población objetivo, de modo de resolver un problema o atender a una necesidad que la afecte". En este caso el Estado de Chile se ha preocupado por establecer cursos de acción, para responder a las necesidades del segmento de personas 
mayores de 60 años, con el propósito específico de generar condiciones favorables para el envejecimiento. Es así que desde el año 1995 ha implementado políticas públicas destinadas para este sector, las cuales poseen objetivos específicos, donde ha implementado medidas permanentes para cumplirlos.

\section{Contexto general}

La vejez es una condición que ha sido analizada históricamente. Algunas posturas de distintos estudiosos de la materia señalan la importancia que tiene esta etapa de la vida en la sociedad. Es evidente que el envejecimiento de la población, además de ser un gran logro de la sociedad, se ha transformado en un gran desafío para la comunidad mundial, especialmente por el impacto que tiene en la carga financiera de los respectivos países.

Una mirada al proceso de envejecimiento — desde la antropología- posibilita identificar factores que permiten que esta etapa de la vida sea una experiencia más llevadera. La importancia de la condición de salud/enfermedad, la trascendencia de una condición económica estable, la relevancia del vivir y del morir acompañado, la muy diferente experiencia de la vejez según la condición de género y la creciente necesidad de que el envejecimiento sea considerado una responsabilidad social ante el escaso apoyo institucional, entre otros (Siller y Serrano, 2006).

Con el paso del tiempo, la esperanza de vida ha ido en aumento, causando un crecimiento en la población mayor de 65 años, lo cual la transforma en objeto de preocupación. En una sociedad como la actual, donde se busca generar condiciones de igualdad para todos quienes habitan un lugar, independiente de su condición económica, debe existir un mínimo esperable el cual debe ser asegurado y resguardado por el Estado, entendiendo que todas las personas tienen derechos, así como obligaciones.

A nivel mundial, el proceso de envejecimiento de la población ha mostrado una constante tendencia al alza en los últimos 30 años, aumentando rápida y progresivamente el número y la proporción de personas mayores de 65 años de edad.

Según los estudios de la CIEDESS (2012), se proyecta que la población con más de 65 años pasará desde cerca de $10 \%$ hasta poco menos de $15 \%$ en el 2025, y a más de 20\% hacia el 2050. Mirado desde el punto de vista de porcentaje sobre la población económicamente activa, pasaría desde poco más de $10 \%$ hasta aproximadamente $30 \%$ hacia el 2050. 
Por su parte, los estudios realizados por el Programa de las Naciones Unidas para el Desarrollo (PNUD, 2013) señalan que entre 1970 y 2011 la población mundial creció de 3,6 mil millones a 7 mil millones de habitantes. El diseño de políticas públicas se ve influido por la estructura etaria de la población, así como también por su tamaño.

El envejecimiento de la población no es algo nuevo en el mundo, quizás para Latinoamérica la toma de conciencia de este fenómeno es un proceso que de a poco se ha ido instaurando en sus respectivos países, pero en Europa, por ejemplo, llevan mucho camino recorrido y se han definido ciertas alternativas posibles de solución, donde sin duda el Estado ha desempeñado un rol protagónico en la definición de políticas específicas sobre estos tópicos.

\section{Envejecimiento de la población en países de Latinoamérica}

En América Latina, según estudios realizados por el Centro Latinoamericano y Caribeño de Demografía (Celade), en el periodo 1970 y 2010, la población en la región creció de 277,7 millones a 577,3 millones de habitantes, generando una proyección hasta el año 2100 de 646,7 millones de habitantes.

El mismo estudio señala que los cambios demográficos afectarán profundamente a la mayoría de los países en las próximas décadas, pero de modos muy diferentes. Las poblaciones ahora envejecen más rápidamente que en el pasado, ya que las tasas de fecundidad bajan y la esperanza de vida aumenta (Banco Mundial, 2013).

Según las estadísticas publicadas por la Comisión Económica para América Latina y el Caribe (CEPAL, 2012), en su respectivo anuario, se está produciendo una baja sostenida del número de hijos por mujer; en promedio ha disminuido de siete hijos por mujer, que era en el quinquenio de $1950 \mathrm{a}$ 1955, a dos o uno desde el año 2010.

Complementando lo anterior, la esperanza de vida en países de Latinoamérica se ha incrementando considerablemente, es decir, de 50 años que era en la década de 1950 a 1960, ha aumentado en promedio a los 75 años, y se proyecta que en el año 2050 la esperanza de vida sea más de 80 años en promedio.

El envejecimiento está avanzando más rápido aún en los países en desarrollo. En ocho, de una muestra de nueve países en desarrollo, se prevé que el porcentaje de la población de mayor edad alcanzará el 14\% en 30 años o menos (PNUD, 2013: 101). 
El índice de envejecimiento poblacional que expresa la relación entre la cantidad de personas adultas mayores y la cantidad de niños y jóvenes es relevante y marca una evidencia más de lo que los países de América Latina están viviendo en relación con la composición etaria de su población.

La forma de cálculo realizada por Celade es el cociente entre personas de 60 años y más respecto a las personas entre 0 y 14 años, multiplicado por 100 . Esto significa, por ejemplo, que un valor de 10 implica que hay 10 adultos mayores (de 60 años y más) por cada 100 niños y jóvenes (menores de 14 años).

Según su interpretación convencional, se trata de un indicador asociado a las transferencias intergeneracionales, y su aumento sistemático implica para los Estados una mayor inversión en salud y seguridad social orientada a las personas de edad (Celade, 2013).

Los resultados que arroja este indicador son fundamentales al momento de tomar decisiones en relación con los programas y políticas que los Estados deben definir; algunos ejemplos de estos resultados en países de Latinoamérica señalan que el índice de envejecimiento va en ascendencia. En Chile aumentó de 30 adultos mayores por cada 100 niños y jóvenes en el año 1990, a 59,1 en el año 2010. El caso de México en el mismo periodo sube de 16,8 a 33,1 adultos mayores por cada 100 niños y jóvenes. Se destaca el caso de Uruguay, que en el año 2010 alcanza a 82 adultos mayores por cada 100 niños menores de 14 años (véase Gráfico núm. 1). ${ }^{1}$

Complementando lo anterior, David Cornejo (2013) afirma que por décadas la región - América Latina - se caracterizó por altas tasas de natalidad, familias numerosas y un excedente de mano de obra. Sin embargo, está transformando su demografía, con una tasa de natalidad que cae cada año y un aumento de la esperanza de vida. De acuerdo con cifras de la ONU, el porcentaje de población de más de 65 años se triplicará para mediados de este siglo, superando el $20 \%$ de la población total.

A su vez, las cifras proyectadas por la CEPAL (2012) indican que la población de más de 65 años en América Latina ascenderá de 6,9\% de la población total en el 2010 a un 19,3\% en el año 2050, y la población de 0 a 14 años descenderá de $27,7 \%$ de la población total en el 2010 a un $17,2 \%$ en el año 2050.

Esta situación ha hecho que todos los Estados busquen nuevas alternativas para enfrentar el desafío del envejecimiento de la población y de los cambios demográficos en América Latina, en ámbitos como salud, seguridad social, educación, vivienda, entre otros.

1 Este gráfico y la tabla se encuentran al final del presente artículo, en el Anexo. 


\section{Envejecimiento de la población en Chile}

El envejecimiento en Chile no se aleja de la realidad mundial, las proyecciones cuantitativas de la población, realizadas por entidades nacionales como el Instituto Nacional de Estadísticas (INE) e internacionales como el Celade, reflejan y proyectan resultados que permiten al Estado planificar y construir políticas públicas que respondan a las necesidades de la población de hoy y de mañana.

Estudios llevados a cabo por las entidades antes mencionadas señalan que en el año 2005 la población del país habría llegado a los 16.267.278 habitantes. Se pronostica que para el año 2020 aumente a 17.865 .000 habitantes (INE, 2005).

Otro indicador que refleja el envejecimiento de la población es el Índice de Adulto Mayor (IAM), que se define como el número de adultos mayores por cada cien menores de 15 años. Corresponde al cociente entre la población de 60 años o más y la población menor de 15 años (INE, 2008).

En el Censo del año 2012 en Chile, el INE señala que el IAM asciende a 67,14 adultos mayores -de 60 años o más-por cada 100 menores de 15 años, lo cual evidencia que este indicador continúa creciendo si lo comparamos con el 59,1 del año 2010 calculado por el Celade.

El INE indica, además, que la distribución por grupos de edad ha variado significativamente desde mediados del siglo pasado. En 1950 los menores de 15 años representaban el 36,7\% de la población total, los de 15 a 64 años eran el 59\% y los mayores de 65 años alcanzaban al 4,3\%. Se estima que al final del periodo de la proyección, 2050, estos grupos serán: $16,6 \%, 61,8 \%$ y $21,6 \%$ lo cual supone un continuo envejecimiento de la población chilena (INE, 2012).

Al observar las proyecciones de la población en Chile, se puede evidenciar que el envejecimiento de la población es una variable con la que el Estado debe trabajar, no puede desatenderla pues las consecuencias podrían ser de suma trascendencia, especialmente en cuanto al cumplimiento de los objetivos de éste, en particular lo relacionado con el bienestar de la población.

Frente a esto, internacional como nacionalmente, se han desarrollado diversas vías de acción que permitan palear los efectos de este problema en desarrollo, se crean planes, proyectos, programas, incluso políticas para poder dar respuesta a esta necesidad urgente y buscar mejorar las condiciones de vida de la población mayor. En Chile, se han creado ciertos mecanismos que permiten direccionar la acción del Estado hacia la generación de un entorno favorable, considerando la mayor cantidad de sectores posibles, para quienes ingresan a la vejez. 


\section{Acciones del Estado para enfrentar el nuevo escenario}

En los últimos años, el Estado de Chile se ha preocupado por buscar alternativas de políticas públicas para el segmento de personas mayores de 60 años, comúnmente denominados "adultos mayores"; para ello, se han desarrollado algunas acciones por medio de las cuales se espera generar condiciones favorables para el envejecimiento.

Las primeras aproximaciones del Estado de Chile a este nuevo escenario comienzan a partir del año 1995 con la creación de la Comisión Nacional para el Adulto Mayor, órgano asesor del Presidente de la República. Al siguiente año, en 1996, se aprueba la Política Nacional para el Adulto Mayor, posteriormente se crean a través del Decreto Supremo núm. 9, de 1997, del Ministerio Secretaría General de la Presidencia de la República de Chile, los Comités Regionales para el Adulto Mayor considerándolos como comités operativos del Servicio.

Un hito importante surge en 1999, año internacional del adulto mayor y en el cual se envía al Congreso Nacional el proyecto de ley que crea el Servicio Nacional del Adulto Mayor (Senama).

En julio de 2002, el Congreso Nacional aprueba el proyecto de ley de la creación del Senama, bajo la promulgación de la Ley núm. 19.828/2002; luego de diez años de funcionamiento de esta institucionalidad, se crea la "Política Integral de Envejecimiento Positivo para Chile 2012-2025".

Paralelo a lo anterior, el Estado ha diseñado e implementado programas y beneficios dirigidos a responder a las necesidades del adulto mayor, en los diversos ámbitos que permitan mejorar su calidad de vida, su bienestar económico, social y cultural.

El Servicio Nacional del Adulto Mayor, de acuerdo con la ley, tiene por objeto velar por la plena integración de estas personas a la sociedad, su protección ante el abandono e indigencia, y el ejercicio de los derechos que la Constitución de la República y las leyes le reconocen.

Este mismo servicio, según el artículo núm. 3 de la Ley núm. 19.828/2002, se encargará de proponer las políticas destinadas a lograr la integración familiar y social efectiva del adulto mayor y la solución de los problemas que lo afectan.

Como hito más próximo se encuentra la Política Integral de Envejecimiento Positivo, que

constituye un avance sustancial que recoge cabalmente los desafíos del envejecimiento, que permite proteger la salud funcional de las personas mientras van envejeciendo, frena el avance de los niveles de dependencia, fomenta el autocuidado, la identidad, la auto- 
nomía y la participación de las personas mayores, intenta terminar con la exclusión y el maltrato y fomenta la integración social, económica y cultural desde una perspectiva de sujetos de derechos. (Senama, 2012:3) .

A partir de aquello, la Política Integral de Envejecimiento se centra en tres aspectos fundamentales: proteger la salud funcional de las personas mayores, mejorar su integración y participación en distintos ámbitos de la sociedad e incrementar de forma transversal el bienestar subjetivo de las personas mayores.

La política citada se sustenta en diversas áreas que buscan "enfrentar el problema del envejecimiento y la vejez y que, más allá de limitarse a solucionar problemas, se adelanta a los acontecimientos, enfrenta los desafíos actuales y prepara a Chile para el futuro" (Senama, 2012: 2).

Las áreas que abarca la Política Integral de Envejecimiento están compuestas por: el área de turismo, salud, trabajo, educación, ingresos familiares, vivienda, cultura, transporte, participación social y justicia.

Además de las diez áreas prioritarias, esta política propone 13 objetivos específicos, los cuales se dan a conocer en la Tabla 1.

Al año 2013 Chile ha implementado, por medio de sus Ministerios y Servicios Públicos, programas y beneficios dedicados al adulto mayor. A continuación se muestra un análisis por objetivo que refleja la coherencia existente entre éstos y los programas y beneficios desarrollados por los distintos Ministerios del Estado.

\section{Respuesta del Estado de Chile a los objetivos específicos de la Política Integral de Envejecimiento 2012-2025}

El objetivo núm. 1: Mejorar la oferta, calidad y eficiencia tanto de los servicios de cuidado como de protección, prevención, atención y rehabilitación de la salud de las personas mayores. Se responde principalmente por medio de programas ejecutados por el Ministerio de Salud, como el Programa Salud del Adulto Mayor, el Plan de Garantías Explícitas de Salud, Examen Preventivo para Personas Mayores, Controles de Salud, el Programa de Rehabilitación con Base Comunitaria, el Programa de Enfermedades Respiratorias, Programa Salud Cardiovascular, Programa Alimentación Complementaria del Adulto Mayor, Programa de Inmunizaciones, Programa Adulto Mayor en Movimiento y el Programa de Atención Domiciliaria para Personas con Dependencia Severa. Estos programas, en conjunto, buscan mejorar las condiciones de salud de las personas mayores mediante servicios eficientes de atención, prevención, protección, permitiendo acompañar el proceso de envejecimiento de éstas. 
Asimismo, el Ministerio de Desarrollo Social, mediante sus programas: Fondo Nacional del Adulto Mayor y el Programa Vínculos, buscan mejorar las condiciones de vida de las personas, tanto en lo que respecta a su cuidado mediante el aporte de este fondo de instituciones intermedias que se hacen cargo de esto, como a su salud psicológica, acompanándolos e insertándolos en la sociedad.

Por otra parte, el Ministerio de la Secretaría General de Gobierno, por medio del programa Adulto Mayor en Movimiento, junto con el Gobierno de Chile y su Programa Elige Vivir Sano, buscan contribuir a la mejora de la calidad de vida de las personas mayores, cuidando aspectos fundamentales como son la alimentación y la actividad física, previniendo enfermedades propias de una vida no saludable.

El objetivo núm. 2: Aumentar la prevalencia de factores protectores para la salud en las personas mayores. Se da respuesta con los mismos programas del Ministerio de Salud del objetivo anterior, junto con un programa del Ministerio de Desarrollo Social, programa Vínculos, el cual apoya psicosocialmente a las personas mayores en situación de vulnerabilidad social para que éstas generen redes sociales. Agregándose la participación del Ministerio de Economía, Fomento y Turismo con el programa Vacaciones Tercera Edad, que posibilita a los adultos mayores mantenerse activos, mediante el fomento de la recreación, permitiendo cuidar la salud física y mental. A su vez, el Ministerio Secretaría General de Gobierno con el Programa Adulto Mayor en Movimiento junto con el Programa de Gobierno Elige Vivir Sano pretenden fomentar una mejor calidad de vida con la actividad física y alimentación sana, factores claves para una buena salud.

El objetivo núm. 3: Aumentar el número de profesionales y técnicos con conocimientos específicos sobre personas mayores. Lamentablemente no presenta relación con ninguno de los programas actualmente vigentes, demostrando una falencia en cuanto a la capacidad de atención especializada disponible. Tanto es así que a finales del año 2012 existían 32 médicos geriatras inscritos en el Fondo Nacional de Salud (Fonasa, 2012).

El objetivo núm. 4: Aumentar las oportunidades que tienen las personas mayores para participar en actividades sociales, recreativas y productivas. Tiene respuesta por medio de cuatro programas del Ministerio de Desarrollo Social:

- En primer lugar, el programa Turismo Social permite dar oportunidades a las personas mayores para que conozcan lugares de interés histórico, cultural y turístico. Este programa aporta significativamente a la recreación e integración social, fortaleciendo las redes de apoyo de estas personas. 
- En segundo lugar, el programa Asesores Seniors busca relacionar a los adultos mayores con niños y niñas pertenecientes a familias vulnerables apoyándolos para mejorar su rendimiento escolar, de forma que ellos se integren activamente a la sociedad, sintiéndose un aporte para ella.

- En tercer lugar, el programa Vínculos, mencionado anteriormente, aporta al cumplimiento de este objetivo mediante la preparación y apoyo psicosocial de las personas mayores para integrarse en actividades sociales.

- En cuarto lugar, el programa Escuela Formación de Dirigentes Mayores tributa a este objetivo entregando herramientas para la formación de líderes mayores, posibilitando la inclusión y participación activa de este grupo etario.

Además de lo anterior, el Ministerio de Educación aporta a este objetivo mediante su programa Estudios Modalidad Flexible, el cual genera oportunidades para que los adultos mayores terminen sus estudios básicos y/o medios, lo que contribuye a su productividad.

Otro de los ministerios que da respuesta a este objetivo es el de Economía, Fomento y Turismo, con su programa Vacaciones Tercera Edad, el cual permite acercar a este sector de personas a actividades recreativas ligadas al turismo, integrándolas socialmente, creando y fortaleciendo vínculos importantes entre ellas. El Ministerio Secretaría General de Gobierno, en conjunto con el Ministerio de Salud - con su programa Adulto Mayor en Movimiento - también contribuye a entregar oportunidades de recreación para las personas mayores, mediante talleres y eventos los cuales se realizan a lo largo del año.

Y en último lugar, se encuentra el programa de gobierno Elige Vivir Sano, el cual promueve actividades recreativas al aire libre y la vida en familia, integrando activamente a la sociedad a los adultos mayores.

El objetivo núm. 5: Mejorar el nivel educacional y formación laboral de las personas mayores. Se responde por medio de dos programas:

- En primer lugar, el del Ministerio de Desarrollo Social, escuela de formación de dirigentes mayores, que entrega herramientas de formación sindical, contribuyendo a la integración laboral de los adultos mayores.

- En segundo lugar, a través del Ministerio de Educación, se imparte el programa Estudios modalidad flexible, el cual contribuye a completar el nivel educacional de este grupo etario. 
El objetivo núm. 6: Proteger la seguridad económica de las personas mayores. A diferencia del resto de los objetivos, se responde por medio de seis beneficios impartidos por el Ministerio de Trabajo y Previsión Social:

- El primer beneficio corresponde al bono bodas de oro que se entrega a las parejas que hayan cumplido 50 años de matrimonio, buscando premiar la institución de la familia como base de nuestra sociedad.

- En segundo lugar, se encuentra el bono por hijo, el cual tiene por objeto mejorar el monto final de la pensión de las mujeres mayores de 65 años de edad.

- En tercer lugar, el bono de invierno tiene como finalidad poder ayudar a enfrentar los gastos propios de la época invernal de los adultos mayores pensionados de menores ingresos.

- En cuarto lugar, el beneficio de la eliminación o rebaja del 7 al 5\% de salud permite resguardar el bienestar económico de las personas mayores evitando disminuir los ingresos de estos.

- En quinto lugar, se encuentra la pensión básica solidaria de vejez, la cual permite que personas que no tienen derecho a la pensión en ningún régimen previsional obtengan un ingreso que les posibilite subsistir.

- Y en última instancia, el aporte previsional solidario de vejez tiene como propósito incrementar las pensiones que se encuentran bajo el monto fijado en la ley que rige este beneficio (Ministerio del Trabajo, 2008).

En suma, los beneficios mencionados anteriormente apuntan a contribuir al mejoramiento de las condiciones económicas con las cuales las personas mayores financian sus necesidades, permitiéndoles tener una mejor capacidad adquisitiva. Fundamental en este sentido han sido la eliminación o rebaja de descuentos de salud, y los aportes previsionales, mejorando la base del ingreso familiar.

El objetivo núm. 7: Adecuar viviendas, medios de transporte y ciudades para las personas mayores. Es respondido por el Ministerio de Vivienda y Urbanismo, mediante su Programa Fondo de Servicios de Atención del Adulto Mayor, el cual busca soluciones habitacionales para personas mayores en situación de vulnerabilidad con tres tipos de instrumentos: establecimientos de larga estadía, conjunto de viviendas tuteladas y viviendas stock. De esta forma se pretende mejorar las condiciones de vivienda y habitabilidad de las personas mayores, adecuando los espacios a sus necesidades, además de la prestación de servicios de apoyo a éstas, de acuerdo con su grado de dependencia. 
Respecto del objetivo que busca adecuar medios de transporte y ciudades para las personas mayores, no se aprecian programas que respondan concretamente a dichas tareas.

El objetivo núm. 8: Disminuir la prevalencia del maltrato activo o pasivo contra las personas mayores. Es respondido a través del programa del Ministerio de Desarrollo Social, Contra la Violencia y el Abuso al Adulto Mayor, que ha permitido incorporar dentro del marco normativo respecto de la violencia al adulto mayor identificándolo como sujeto de derecho, especializando la atención otorgada a ellos en el caso de violencia activa y/o pasiva. Además de lo anterior, permite sensibilizar a la ciudadanía frente a este tipo de hechos.

El objetivo núm. 9: Aumentar el acceso a la justicia que tienen las personas mayores como titulares de derechos. Al igual que el objetivo anterior, se da respuesta por medio del Programa Contra la Violencia y el Abuso al Adulto Mayor, mediante el apoyo y asesoría legal que otorga el Servicio Nacional del Adulto Mayor a las personas mayores que presenten algún tipo de problema de esta índole, mejorando su acceso a la defensa de sus derechos.

El objetivo núm. 10: Mejorar la cobertura y calidad de los servicios estatales de atención de consultas y difusión de información relacionada con personas mayores. Se responde a través del Programa del Ministerio de la Secretaría General de la Presidencia "Chile Atiende", que permite acercar los distintos servicios del Estado a las personas, evitando largos tramos de desplazamiento para dar respuesta a sus necesidades en cuanto a su relación con éste. Además, permite difundir información relevante de los servicios y beneficios que se entregan a las personas mayores.

El objetivo núm. 11: Potenciar una identidad social y cultural positiva de las personas mayores. Es respondido por dos programas, dependiendo el primero Programa Asesores Seniors del Ministerio de Desarrollo Social, el cual contribuye a que las personas mayores se identifiquen como actores sociales importantes, ya que cumplen una labor social ayudando a niños con dificultades de aprendizaje. Por otra parte, el Programa Turismo Social depende del mismo ministerio y contribuye a la integración social de las personas mayores en situación de vulnerabilidad, acercándolas a la cultura, historia y lugares característicos del país, identificándolas con sus orígenes culturales.

El objetivo núm. 12: Aumentar el número de investigaciones en temas relacionados con el envejecimiento y vejez. No ha tenido cumplimiento por parte del Estado chileno, posiblemente por falta de incentivos, sean éstos académicos, monetarios u otros que permitan desarrollar este polo de investigación, que ha tomado especial relevancia en los últimos años como preocupación pública. 
El objetivo núm. 13: Evaluar y optimizar periódicamente la contribución de la Política al bienestar subjetivo de las personas mayores. No ha logrado un desarrollo demostrable, por lo menos no se observa en fuentes oficiales, programas o beneficios asociados a dichas metas. Da la impresión de que falta generar evaluaciones ex post, que midan el impacto que han tenido las medidas desarrolladas pre y post política pública en cuanto al envejecimiento, pues permitirá tomar medidas correctivas respecto de aquellas áreas donde no se obtengan los resultados esperados.

A modo de corolario, se podría afirmar que debe considerarse que en el cumplimiento de los objetivos no sólo hay tópicos sin desarrollar, pues hay algunos aspectos en los cuales se han concentrado los esfuerzos, principalmente en lo relacionado con salud e inserción social, viéndose reflejado en un alto número de programas y/o beneficios que apuntan a mejorar las condiciones del sector salud en cuanto a oferta, calidad y eficiencia y al aumento de actividades que permiten que los adultos mayores participen de la vida pública.

Para reafirmar lo anterior, la Encuesta de Calidad de Vida en la Vejez muestra antecedentes importantes al momento de diseñar nuevas iniciativas y políticas públicas que busquen responder a los desafíos y necesidades que surgen de la realidad de los adultos mayores.

Los resultados de la tercera Encuesta de Calidad de Vida en la Vejez 2013 muestran un alza sostenida de la satisfacción general con la vida: pasando de un 56,2\% en el año 2007, a un 59,5\% en el año 2011, y a un 62,8\% en 2013. El estudio señala que esta ascendencia puede ser consecuencia de la mejora en las condiciones de vivienda y al incremento en la satisfacción de las necesidades de afecto y recreación de los encuestados, lo cual refuerza la Política de Envejecimiento Activo.

Otro factor más específico que considera esta encuesta y que arrojó resultados positivos es el porcentaje de personas mayores que prefieren quedarse en casa en vez de salir y hacer cosas nuevas; muestra que entre 2010 y 2013 bajó desde un 60 hasta un 53\%; esto podría ser el reflejo del aumento de diferentes actividades recreativas, que han sido contempladas en los programas de Gobierno a través de sus Ministerios.

Uno de los puntos que se ha mantenido en una de las crecientes preocupaciones del adulto mayor es la Salud, los resultados de la encuesta señalan que cerca del $90 \%$ de los encuestados reconoce poseer al menos una enfermedad, predominando aquellas relacionadas con la hipertensión, colesterol alto o diabetes, las cuales reflejan un aumento sostenido en su prevalencia conforme a lo manifestado por las personas mayores encuestadas desde 2007 hasta 2013. En esta misma línea, la encuesta arroja un alarmante resultado; 
éste corresponde al aumento en la ingesta de medicamentos, que pasó de 2,9 en promedio el año 2007 a 3,6 para 2013, precisando que la mayor ingesta de medicamentos se da entre mujeres, personas de más edad y personas sin educación superior.

Asimismo, otro de los elementos donde hay constante preocupación es en lo que respecta a proteger la seguridad económica de las personas, buscando las medidas necesarias para poder generar condiciones favorables de desarrollo, principalmente en lo previsional.

Por lo anterior, conocer los resultados de esta encuesta en relación con el nivel económico es primordial, y los resultados señalan que cerca de un $28 \%$ de los encuestados realizó algún trabajo remunerado en el último mes, a pesar de que, en su mayoría, los ingresos percibidos por las personas mayores les alcanzan al justo para cubrir sus gastos o, simplemente, no les alcanzan.

Complementando lo anterior, el 20,8\% de las personas mayores declara tener la necesidad de capacitarse para mantenerse o volver al trabajo, pero sólo el 8,3\% declara haber recibido algún tipo de formación en los últimos años.

También se debe reconocer que el actual sistema de pensiones en Chile es insuficiente, lo cual se traduce en una tasa de reemplazo de las remuneraciones al momento de jubilar, que no alcanza los niveles esperados por las personas mayores.

$\mathrm{Al}$ año 2014, son diez millones los afiliados al sistema de pensiones de Chile, capitalización individual, de los cuales 4,7 millones son cotizantes activos, y de éstos sólo 400 mil tienen ingresos iguales o mayores al tope imponible.

El 60\% de los cotizantes activos desde 1981 posee saldos menores a los 31.012,56 dólares (20 millones de pesos chilenos), por lo que alcanzan una pensión de 232,594 dólares (150 mil pesos chilenos); ante ello, se ven obligados a utilizar el beneficio del "aporte previsional solidario", para alcanzar la pensión mínima garantizada por el Estado.

Estudios desarrollados por la Organización para la Cooperación y el Desarrollo Económicos (OCDE, 2013) reafirman que no se alcanza el 70\% de la tasa de reemplazo que comprometía el sistema de pensiones de Chile, y señalan que un afiliado hombre, quien se pensiona a los 65 años de edad, que comenzó a cotizar a los 25 años de edad, cuyo salario real creció al 1,5\% anual durante su vida, recibirá una pensión líquida igual al 54\% del promedio de los tres últimos años de salario líquido. En el caso de la mujer, el escenario empeora, pues la legislación vigente señala que se debe jubilar a los 60 años de edad, por lo tanto, en el caso de que comience a cotizar a los 25 años de edad, cuyo salario también crece al 1,5\% anual, recibirá una pensión líquida igual al $39 \%$ del promedio de los tres últimos años de salario líquido. 
Los ejemplos anteriores reflejan los mejores escenarios en los resultados de la tasa de reemplazo, pues considera que las personas cotizan durante todos los años de su vida laboralmente activa; pero la realidad de Chile genera una mayor preocupación: a septiembre del año 2013, del total de afiliados, 2,2 millones poseen lagunas en sus cotizaciones entre 0 a 5 años, 1,1 millones no cotiza entre 5 a 14 años, y 1 millón de afiliados posee lagunas de más de 14 años; las razones son por cesantía o por su tipo de contrato. Por lo tanto, los hombres terminan cotizando en promedio 21,5 años para financiar 20 años, y las mujeres cotizan en promedio 15 años para financiar pensiones de 30 años.

Lo anterior demuestra la disminución real en la calidad de vida de las personas al momento de jubilarse, lo cual refuerza el rol del Estado en seguir implementado programas que permitan solventar en parte este descenso.

Lo positivo de las preocupaciones manifestadas es que estas problemáticas, que han permanecido en el tiempo y que se espera puedan incrementarse por el aumento de la población de tercera edad, se encuentran contenidas en la Política Integral de Envejecimiento Positivo y forman parte del programa del actual gobierno, quien por medio del Servicio Nacional del Adulto Mayor consideró pertinente dejar de utilizar el concepto de envejecimiento positivo y trabajar con los conceptos de envejecimiento activo, basado en un enfoque transversal de derechos, desde donde se llevan a cabo acciones, planes y programas definidos, que promueven la articulación de la oferta pública para las personas mayores, sin dejar de lado los ámbitos de la participación y fomento del envejecimiento activo, donde existe un mayor desarrollo y avance.

\section{Conclusiones}

El Estado chileno ha generado avances respecto a la preparación y protección, frente al desafío del envejecimiento que está viviendo su población, se han diseñado lineamientos necesarios para poder enfrentar de la mejor manera posible dicho proceso, considerando aquellos aspectos ineludibles en la búsqueda de mejorar las condiciones de vida de las personas mayores. Frente a esta realidad quedan muchas tareas que asumir en los distintos niveles y sectores que este tema comprende.

Por una parte, para la seguridad social, el envejecimiento poblacional es el desafío más importante que plantean los cambios demográficos. Es preciso recalcar que el "envejecimiento" no sólo hace referencia a la creciente proporción de ciudadanos mayores, sino más que nada a un desequilibrio en los grupos de edades, una disminución de la población más joven, debido 
al decrecimiento de la tasa de natalidad, un aumento de la población activa, que acabará disminuyendo en un futuro bastante cercano o no -según sea el país que se analice- y por supuesto, un creciente número de personas mayores y muy mayores, que tal vez necesiten ayuda en función de su estado de salud.

Otro de los desafíos a los que se enfrenta Chile en el mediano plazo apunta a mejorar la calidad de vida de las personas mayores, buscando opciones para que éstas generen más ingresos mediante la inserción al mercado laboral, con condiciones laborales acordes a los índices de calidad de vida esperados y que permitan soslayar la deficiencia previsional de la vejez.

Por otra parte, asumiendo también que la calidad de vida depende totalmente de la calidad de las prestaciones de salud que se entreguen a las personas, es un verdadero reto para el Estado chileno conseguir la adaptación temprana y concreta de los sistemas de salud a los cambios de demanda propios de la dinámica demográfica actual y que va en constante aumento. Esto significa, además, mejorar la cobertura de atención sanitaria para que permita entregar las condiciones, tanto profesionales como de infraestructura, hacia una atención de calidad.

Otro aspecto a considerar -dentro de las tareas por concretar por parte del Estado chileno - es la falta de proactividad en cuanto a la protección y defensa de los derechos en la vejez, entendiendo que aún existen ciertos atisbos de discriminación hacia las personas en función de su edad. Cuestiones críticas frente a esta vulnerabilidad son el difícil acceso a servicios básicos como vivienda, educación, entre otros.

Otro desafío tiene que ver con la falta de eficiencia que ha mostrado el sistema previsional de capitalización individual, el cual no ha sido capaz de responder eficazmente a las necesidades de calidad de vida de las personas luego de su jubilación, haciendo que adultos mayores terminen en condiciones de vida no adecuadas.

Dentro de la búsqueda de opciones que permitan mejorar las condiciones de vida de los adultos mayores se debe considerar como un factor relevante -en cuanto a la generación de lineamientos de acción desde el Estado-: evitar la implementación inmediata de modelos internacionales que se hayan generado en respuesta a esta problemática. Lo anterior implica suponer que es necesario considerar previamente la adecuación de dichos modelos a las distintas realidades y contextos que se desarrollan dentro del país correspondiente.

Por otra parte, la educación y capacitación constante es otro de los puntos a los cuales el país debe apuntar; es tarea del Estado preparar a su población adulta para poder insertarse o mantenerse de mejor forma dentro del mercado laboral activo. A su vez, es imperioso que la educación (en materias de previ- 
sión y vejez) se imparta desde temprana edad, pues permitirá mitigar de mejor forma los efectos adversos (en el plano del empleo y la falta de recursos) que se van produciendo, en la mayoría de los casos, con el paso de los años, preparando a la población y mejorando su calidad de vida.

Si bien es cierto existe en Chile una política pública que busca mejorar las condiciones de vida de las personas mayores, falta aún por avanzar en la concreción de dichos lineamientos. Es necesario fortalecer y desarrollar aún más la preocupación del Estado frente a las nuevas necesidades que aparecerán en el mediano y largo plazo, especialmente en el desarrollo integral de los adultos mayores. Respecto a los objetivos que define esta política, se puede observar coherencia entre lo que ahí se plantea con los programas, servicios y beneficios que el Estado ha entregado desde que el tema comienza a formar parte de la preocupación y discusión pública. Pero quedan puntos por resolver, especialmente los relacionados con la efectiva concreción de las ideas y la generación de un impacto positivo tanto en la población, en su totalidad, como en la población mayor. Por ejemplo, uno de los aspectos que se no se ha logrado visibilizar aún es el maltrato hacia los adultos mayores, del cual no se ha logrado obtener información suficiente, por eso las acciones correctivas en este sentido han sido escasas y no se ha podido instalar el problema dentro de la opinión pública, dejándolos de cierta forma, en un estado de indefensión constante.

La falta de incentivos en cuanto a generación y captación de nuevos profesionales especialistas en el área es un aspecto frente al cual se deben tomar medidas en el corto plazo, entendiendo que este es un proceso que seguirá avanzando y trayendo consigo una serie de necesidades que no podrán ser cubiertas si no se cuenta con un número adecuado de expertos en la materia. Adicionado a lo anterior, además de contar con profesionales especializados, es de suma relevancia la generación de conocimiento en la búsqueda de más y mejores soluciones en cuanto al envejecimiento de la población, incentivando y motivando a investigadores de distintas áreas para que integren esta temática dentro de sus preocupaciones, fortaleciendo de esta forma la innovación y el desarrollo de ideas que propendan a un envejecimiento con calidad.

La generación por parte del Estado chileno de una política pública es un paso muy importante para enfrentar este gran desafío, pero si no se lleva a cabo un seguimiento y evaluación de las medidas tomadas, difícilmente se podrá mejorar en cuanto a calidad y efectividad.

Ideas respecto de cómo enfrentar el envejecimiento de la población hay muchas, concretarlas en algunos casos es el problema; por lo mismo, los desafíos que debe enfrentar el Estado chileno son variados, se necesita utilizar el 
tiempo disponible de la mejor manera posible para evitar que este tema pase a transformarse en un problema mayor y de mucho más difícil control.

Lo que está claro es que el país envejece y es misión de todos y cada uno de los chilenos sumar las ideas, el esfuerzo y el compromiso de todos los sectores, para lograr una mejor calidad de vida de sus adultos mayores.

A modo de corolario, podría adicionarse que lo relevante en toda la discusión es que en el Estado chileno exista continuidad en enfrentar los desafíos que buscan responder positivamente a las necesidades de la ciudadanía, y en este caso, el programa impulsado por el Gobierno pretende contribuir al envejecimiento activo de personas mayores en situación de vulnerabilidad social, proporcionando conocimientos y oportunidades para el desarrollo personal, cognitivo, biológico, físico y de participación social, para lo cual se requiere fomentar y equilibrar la responsabilidad personal, el encuentro, la solidaridad intergeneracional y la creación de entornos favorables, que aporten a la calidad de vida y retrasen los niveles de dependencia del adulto mayor.

Por lo anterior, es claro que este tema resulta muy interesante de abordar; por lo mismo, se abren nuevas rutas investigativas que van desde un estudio con fuentes primarias de información, que permitan tener acceso a lo que están pensando y viviendo los adultos mayores, hasta, a su vez, intentar realizar estudios comparativos con otras realidades, tanto de países subdesarrollados, como de aquellos que están en condición de prosperidad.

\section{Bibliografía}

Birkland, T. (2005), An introduction to the policy process: theories, concepts, and models of public policy making, New York: M.E. Sharpe.

Cornejo, David (2013), "Especial pensiones: los modelos necesitan reformas", en Revista América Economía, febrero, Chile: América Economía.

Hernández, Fernández y Pilar Baptista (2006), Metodologia de la Investigación, México: McGraw- Hill.

Jenkins, W. (1978), Policy Analysis: A Political and Organizational Perspective, Londres: M. Robertson.

Kraft, M. y Furlong, S. (2004), Public Policity: Politics, Analysis and Alternatives, Washington DC: CQ Press.

Lahera, E. (2008), Introducción a las Politicas Públicas, Santiago: Fondo de Cultura Económica.

Meny, Y. y J. C. Thoening (1992), Las politicas públicas, Barcelona: Ariel.

Servicio Nacional del Adulto Mayor (2012), "Principales beneficios y programas del Gobierno para las Personas Mayores", en Revista Ser Mayor, año 2012, núm. 6, Chile: Senama.

Siller, Pelcastre y Margarita Serrano (2006), "Experiencias de envejecimiento en el México rural”, en Revista Salud Pública de México „núm. 1, México: Scielo. 
Francisco Ganga-Contreras, María Angélica Piñones-Santana, Diego González-Vásquez y Francisca Rebagliati-Badal. Rol del Estado frente al envejecimiento de la población: el caso de Chile

\section{Recursos electrónicos}

Aguilar, A. y Lima, F. (2009), “Contribuciones a las Ciencias Sociales”. Disponible en: www. eumed.net/rev/cccss/05/aalf.htm [10 de junio de 2013].

CEPAL (Comisión Económica para América Latina y el Caribe) (2012), "Anuario Estadístico de América Latina y el Caribe 2012”. Disponible en: http://www.eclac.cl/ publicaciones/xml/2/48862/AnuarioEstadistico2012.pdf [5 de octubre de 2013].

CIEDESS (Corporación de Investigación, Estudio y Desarrollo de la Seguridad Social) (2012), "Diseño de un Seguro Social de Protección a la Dependencia para Chile. Propuesta de Estudio Santiago”. Disponible en: http://senama.cl/filesapp/Nicolas_ Starck_Seguro_de_Dependencia.pdf[20 de octubre de 2013].

Fonasa (Fondo Nacional de Salud) (2012), "Estadísticas Institucionales". Disponible en: https://www.fonasa.cl/portal_fonasa/site/artic/20140607/pags/20140607230746. html [20 de octubre de 2013].

INE (Instituto Nacional de Estadísticas) (2005), "Chile hacia el 2050: Proyecciones de Población”. Disponible en: http://www.ine.cl/canales/chile_estadistico/ demografia_y_vitales/proyecciones/Informes/Microsoft $\% 20$ Word\%20-\%20 InforP_T.pdf [17 de octubre de 2013].

INE (Instituto Nacional de Estadísticas) (2008), "Población y sociedad, aspectos demográficos”. Disponible en: http://www.ine.cl/canales/chile_estadistico/ demografia_y_vitales/demografia/pdf/poblacion_sociedad_enero09.pdf. $\left[\begin{array}{ll}20 & \text { de }\end{array}\right.$ octubre de 2013].

Ministerio del Trabajo (2008), Ley núm. 20.255 “Establece reforma previsional”. Disponible en: http://www.leychile.cl/Navegar?idLey=20255 [5 de noviembre de 2013].

Ministerio Secretaría General de la Presidencia (2002), Ley núm. 19.828, "Crea el Servicio Nacional del Adulto Mayor”. Disponible en: http://www.leychile.cl/ Navegar ?idNorma $=202950$ [5 de noviembre de 2013].

Ministerio de Planificación (2012), Ley núm. 20.230 "Crea el Ministerio de Desarrollo Social y modifica cuerpos legales que indica”. Disponible en: http://www.leychile.cl/ Navegar idNorma=1030861 [ 10 de diciembre de 2015].

Organización para la Cooperación y el Desarrollo Económicos OCDE (2013). "Pensions at a Glance 2013". Disponible en http://www.oecd-ilibrary.org/docserver/ download $/ 8113221$ e.pdf ?expires $=1459985641 \& \mathrm{id}=\mathrm{id} \&$ accname= guest $\&$ checksu $\mathrm{m}=8 \mathrm{CA}$ 5D79FCE11998037A8BC024AAE464D [30 de agosto de 2015].

Olavarria Gambi, Mauricio (2007). "Conceptos Básicos en el Análisis de Políticas Públicas", en Repositorio Académico de la Universidad de Chile. Disponible en http:// repositorio.uchile.cl/bitstream/handle/2250/123548/Conceptos_\%20Basicos_ Politicas_Publicas.pdf ?sequence $=1$ [30 de mayo de 2013]

PNUD (Programa Naciones Unidas) (2013), "Informe Sobre Desarrollo Humano 2013". Disponible en: http://www.undp.org/content/dam/undp/library/corporate/ HDR/2013GlobalHDR/Spanish/HDR2013\%20Report\%20Spanish.pdf $\quad[17$ de octubre de 2013].

PUC (Pontificia Universidad Católica), Senama y Caja Los Andes (2014), "Chile y sus mayores. Resultados Tercera Encuesta Nacional Calidad de Vida en la Vejez 2013”. Disponible en: http://www.senama.cl/filesapp/Chile\%20y\%20sus\%20mayores\%20 2013,\%20Encuesta\%20de\%20Calidad\%20de\%20Vida.pdf [ 17 de junio de 2014]. 
Senama (Servicio Nacional del Adulto Mayor) (2012),'Política Integral de Envejecimiento Positivo para Chile 2012-2025”. Disponible en: http://www.senama.cl/filesapp/ PIEP-2012-2025.pdf [2 de noviembre de 2013].

SUBDERE (Subsecretaría de Desarrollo Regional y Administrativo) (2009), Guia metodológica para la formulación de politicas públicas regionales. Serie Documentos de Trabajo núm. 5. Disponible en: http://www.subdere.cl/sites/default/noticiasold/ articles-76900_recurso_1.pdf [2 de diciembre de 2013].

\section{Bases de datos}

Banco Mundial, "Base de datos de Indicadores de Desarrollo Mundial". Disponible en: http://datos.bancomundial.org/base-de-datos-actualizada-de-los-Indicadores-deldesarrollo-mundial [20 de noviembre de 2013].

Celade (Centro Latinoamericano y Caribeño de Demografía), "División de la Población de la CEPAL". Disponible en: http://www.cepal.org/celade/proyecciones/basedatos_ BD.htm [13 de noviembre de 2013].

INE (Instituto Nacional de Estadísticas), “Censo 2012”. Disponible en: http://www.ine.cl/ canales/chile_estadistico/familias/censos.php [15 de diciembre de 2013]. 
Francisco Ganga-Contreras, María Angélica Piñones-Santana, Diego González-Vásquez y Francisca Rebagliati-Badal. Rol del Estado frente al envejecimiento de la población: el caso de Chile

\section{Anexo}

\section{Gráfico 1}

\section{Índice de Envejecimiento de la población (por cien) - años 1990-2000-2010}

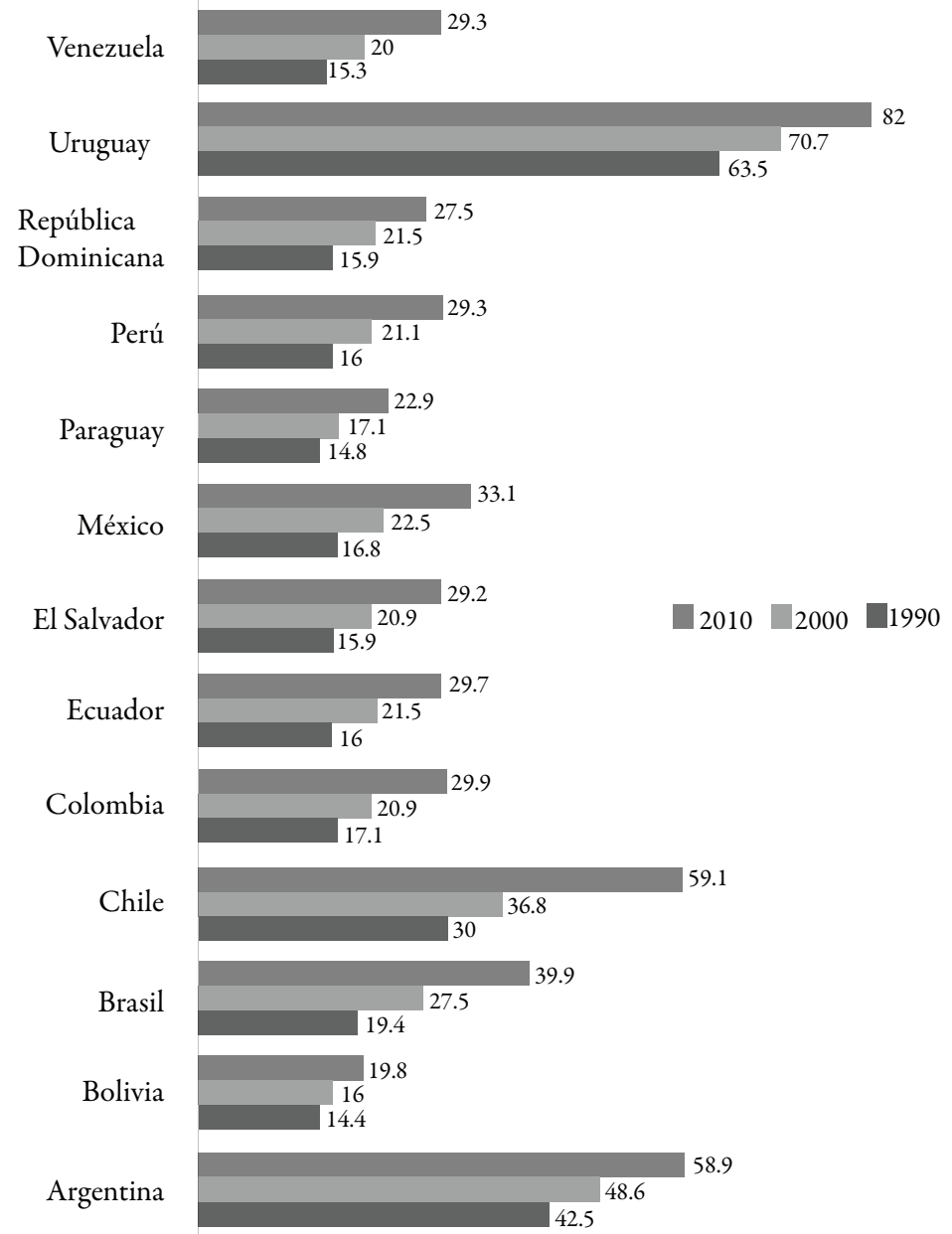

Fuente: Elaboración propia con datos del Centro Latinoamericano y Caribeño de Demografía (Celade) - División de Población de la CEPAL. 


\section{Tabla 1}

\section{Los trece objetivos de la Política Integral de Envejecimiento}

\begin{tabular}{ll}
\hline Objetivo & Descripción del objetivo propuesto \\
\hline Núm. 1 & $\begin{array}{l}\text { Mejorar la oferta, calidad y eficiencia tanto de los servicios de cuidado } \\
\text { como de protección, prevención, atención y rehabilitación de la salud de } \\
\text { las personas mayores. }\end{array}$ \\
\hline Núm. 2 & $\begin{array}{l}\text { Aumentar la prevalencia de factores protectores para la salud en las } \\
\text { personas mayores. }\end{array}$ \\
\hline Núm.3 & $\begin{array}{l}\text { Aumentar el número de profesionales y técnicos con conocimientos } \\
\text { específicos sobre personas mayores. }\end{array}$ \\
\hline Núm.4 & $\begin{array}{l}\text { Aumentar las oportunidades que tienen las personas mayores para parti- } \\
\text { cipar en actividades sociales, recreativas y productivas. }\end{array}$ \\
\hline Núm.5 & Mejorar el nivel educacional y formación laboral de las personas mayores. \\
\hline Núm.6 & Proteger la seguridad económica de las personas mayores. \\
\hline Núm.7 & $\begin{array}{l}\text { Adecuar viviendas, medios de transporte y ciudades para las personas } \\
\text { mayores. }\end{array}$ \\
\hline Núm. 8 & $\begin{array}{l}\text { Disminuir la prevalencia del maltrato activo o pasivo contra las personas } \\
\text { mayores. }\end{array}$ \\
\hline Núm.9 & $\begin{array}{l}\text { Aumentar el acceso a la justicia que tienen las personas mayores como } \\
\text { titulares de derechos. }\end{array}$ \\
\hline Múm. 13 & $\begin{array}{l}\text { Evaluar y optimizar periódicamente la contribución de la Política al } \\
\text { bienestar subjetivo de las personas mayores. }\end{array}$ \\
\hline cimiento y vejez.
\end{tabular}

Fuente: Diseño propio, basado en Senama (2012: 37). 
Francisco Ganga Contreras. Doctor en administración de empresas y postdoctorado en ciencias humanas. Adscrito al Departamento de Ciencias del Desarrollo, Universidad de Los Lagos de Chile-Campus Santiago. Líneas de investigación: gobernanza universitaria, políticas públicas, administración de personal. Publicaciones recientes: Ganga, F.; Ramos, E.; Leal, A. y Valdivieso, P., "Teoría de agencia (TA): supuestos teóricos aplicables a la gestión universitaria”, en Revista Innovar, vol. 25, núm. 57 (2015); Ganga, F.; Ramos, E.; Leal, A. y Valdivieso, P., "Diseño y formulación de un cuadro de mando integral (CMI): Aplicación a una organización no gubernamental", en Revista de Ciencias Sociales, vol. XXI, núm. 1 (2015); Ganga F. y S. Maluk (2015), "Gobierno universitario ecuatoriano: una aproximación teórica a los cambios más relevantes de los últimos años”, en Revista Prisma Social, núm. 14 (2015).

María Angélica Piñones Santana. Doctora en Administración de Empresas de ULSEBT de Bélgica. Académica jornada completa de la Universidad de Valparaíso, Chile. Líneas de investigación:impuestos, políticas públicas, control de gestión, administración pública. Publicación reciente:"Estado e impuestos indirectos en Chile y Latinoamérica: análisis de periodo 2000-2011”, en revista científica Visión de Futuro, Facultad de Ciencias Económicas de la Universidad de Misiones de Argentina (2014).

Diego González Vásquez. Licenciado en Administración Pública de la Universidad de Valparaíso. Académico jornada completa de la Universidad de Valparaíso, Chile. Líneas de investigación:políticas públicas y gestión pública.

Francisca Rebagliati Badal. Licenciada en Administración Pública de la Universidad de Valparaíso. Académica jornada completa de la Universidad de Valparaíso, Chile. Líneas de investigación:políticas públicas y gestión pública.

Recepción: 13 de octubre de 2014.

Aprobación: 11 de enero de 2016. 
